\title{
The correlation of narrow line emission and X-ray luminosity in active galactic nuclei
}

\author{
H. Netzer ${ }^{1,2}$, V. Mainieri ${ }^{2}$, P. Rosati ${ }^{3}$, and B. Trakhtenbrot ${ }^{1}$ \\ 1 School of Physics and Astronomy and the Wise Observatory, The Raymond and Beverly Sackler Faculty of Exact Sciences, \\ Tel-Aviv University, Tel-Aviv 69978, Israel \\ e-mail: netzer@wise.tau.ac.il \\ 2 Max-Planck-Institut für extraterrestrische Physik, Postfach 1312, 85741 Garching, Germany \\ ${ }_{3}^{3}$ European Southern Observatory, Karl-Schwarzschild-Strasse 2, 85748 Garching bei Muenchen, Germany
}

Accepted 14 September 2005 / Accepted 15 March 2006

\section{ABSTRACT}

\begin{abstract}
Aims. We combine emission line and X-ray luminosities for 45 sources from the Chandra Deep Field South (CDF-S), and seven HELLAS sources, to obtain a new sample of $52 \mathrm{X}$-ray selected type-II active galactic nuclei (AGNs). Eighteen of our sources are very luminous with a typical, absorption-corrected 2-10 keV luminosity of $f e w \times 10^{44} \mathrm{erg} \mathrm{s}^{-1}$ (type-II QSOs).

Methods. We compare the emission line properties of the new sources with emission line and X-ray luminosities of known low redshift, mostly lower luminosity AGNs by using a composite spectrum.

Results. We find that $L_{[\mathrm{OIII}]} / L_{2-10}$ and $L_{[\mathrm{OII}]} / L_{2-10}$ decrease with $L(2-10 \mathrm{keV})$ such that $L_{[\mathrm{OIII}]} / L_{2-10} \propto L_{2-10}^{-0.42}$. The trend was already evident, yet neglected in past low redshift samples. This lead to erroneous calibration of the line-to-X-ray luminosity in earlier AGN samples. The analysis of several type-I samples shows the same trend with a similar slope but a median $L_{[\mathrm{OIII}]} / L_{2-10}$ which is larger by a factor of about two compared with optically selected type-II samples. We interpret this shift as due to additional reddening in type-II sources and comment in general on the very large extinction in many type-II objects and the significantly smaller average reddening of the SDSS type-II AGNs. The decrease of $L_{[\mathrm{OIII}]} / L_{2-10}$ with $L(2-10 \mathrm{keV})$ is large enough to suggest that a significant fraction of high luminosity high redshift type-II AGNs have very weak emission lines that may have escaped detection in large samples. A related decrease of $E W([\mathrm{O} \mathrm{III}]$ 25007) with optical continuum luminosity is demonstrated by an analysis of 12000 type-I SDSS AGNs. The new correlations found here are important for deriving accurate luminosity functions for AGNs and their neglect may explain past discrepancies between emission line and X-ray samples.
\end{abstract}

Key words. galaxies: active - galaxies: Seyfert - quasars: emission lines - X-rays: galaxies

\section{Introduction}

The study of the space distribution and the luminosity function (LF) of active galactic nuclei (AGNs) has been the focus of much attention in recent years. In particular, deep X-ray surveys (Hasinger 2004; Ueda et al. 2003, and references therein) have been combined with large ground-based data sets, like the two degree field $(2 \mathrm{dF})$ galaxy redshift survey and the Sloan Digital Sky Survey (SDSS) to study the LFs of type-I and type-II AGNs. Such studies are essential for understanding AGN and galaxy evolution and to explain the contribution of the various sub-groups to the cosmological X-ray background (CXB).

Several extensive studies of the differences between type-I and type-II sources make use of the [O III] $\lambda 5007$ line luminosity. This line originates in the narrow line region (NLR) which is thought to be of much larger dimensions than the putative central torus. Thus, the line emission is considered to be isotropic and to have a similar luminosity distribution in type-I and typeII sources. Work by Mulchaey et al. (1994, hereafter M 94) and Alonso-Herrero et al. (1997, hereafter A97) suggested a simple calibration scheme between the hard (2-10 keV) X-ray luminosity and $L([\mathrm{O} I I I]$ 25007). These papers, and several others since, studied various correlations between $L([\mathrm{O}$ III $]$ 15007), $L(2-10 \mathrm{keV})$ and $L(\mathrm{IR})$ in small samples of mostly low luminosity AGNs. According to these papers, the mean and the distribution of $L([\mathrm{O} \mathrm{III}] \lambda 5007) / L(2-10 \mathrm{keV})$ (hereafter $\left.L_{[\mathrm{OIII}]} / L_{2-10}\right)$ in the two AGN groups is indistinguishable confirming the line isotropy assumption and suggesting that the mean line extinction is also independent of orientation. This M 94 scaling relationship was used as a standard optical-to-Xray conversion tool in numerous other papers, including very recent ones (e.g. Vignali et al. 2004).

More recent studies made use of the large SDSS sample to study the $L([\mathrm{O}$ III $]$ 15007) and the line equivalent width $(E W)$ distribution. Some of the most extensive studies of this type that are relevant to the present work are Zakamska et al. (2003, 2004), Simpson (2005, hereafter S05), Hao et al. (2005) and Heckman et al. (2005). The Zakamska et al. papers made use of the M94 and A97 results and demonstrated the similar distribution of $L([\mathrm{O}$ III $]$ 25007) in the two AGN subgroups in the redshift range $0.3<z<0.8$. They also show an almost perfect linear relationship between $L([\mathrm{O} I \mathrm{II}]$ 23727) and $L([\mathrm{O}$ III $]$ 25007), thus the [O II] $\lambda 3727$ line is also expected to show a luminosity independent ratio with $L(2-10 \mathrm{keV})$, $L([\mathrm{O}$ II $] \lambda 3727) / L(2-10 \mathrm{keV})$ (hereafter $\left.L_{[\mathrm{OII}]} / L_{2-10}\right)$, similar to $L_{[\mathrm{OIII}]} / L_{2-10}$. Heckman et al. (2005) further tested the M 94 assumption and noted the change in the mean $L_{[\mathrm{OIII}]} / L_{2-10}$ between $\mathrm{X}$-ray selected and emission line selected samples. The paper 
also notes the larger range in this property in type-II sources due to X-ray obscuration.

The recent works by S05 and by Hao et al. (2005) focus on line LFs obtained from the SDSS data set. S05 derived [O III] $\lambda 5007$ LFs for the two AGN sub-groups and used the M 94 scaling to compare them with large X-ray samples. He further makes a detailed comparison with the "receding torus" model (see e.g. Grimes et al. 2004; S05). The conclusion is that the model in its simplest form cannot explain the different LFs derived for the two groups. Hao et al. (2005) focus on the similarity of the $\mathrm{H} \alpha$ and [O III] $\lambda 5007$ LFs in two large samples of AGNs with $0<z<0.15$.

Much of the uncertainty in deriving the $L([\mathrm{O}$ III] 25007$) \mathrm{LF}$ for type-II sources is the lack of reliable measurements of this line in high redshift high luminosity sources. There are a handful of notable exceptions (see Stern et al. 2002; Norman et al. 2002) but, so far, the numbers were far from enough to make a detailed study of the population. Almost the only way to cure this deficiency is to combine X-ray fluxes and follow-up spectroscopy in X-ray selected samples. In this paper we adopt this approach and discuss the emission line spectrum of 52 high redshift type-II AGNs, some with very high X-ray luminosity. Most of the sources were discovered in the 1Ms Chandra observation of the Chandra Deep Field South (CDF-S; Giacconi et al. 2002; Rosati et al. 2002) and seven others are obtained from the HELLAS2XMM survey (Fiore et al. 2003). The new sample allows us to address the issue of the observed vs. the expected $L_{[\mathrm{OIII}]} / L_{2-10}$ and $L_{[\mathrm{OII}]} / L_{2-10}$ in type-I and type-II AGNs and to draw conclusions about the LF of type-II sources at high redshifts. Section 2 describes the observations and data sources used in this work and the procedure used for obtaining an "effective $L([\mathrm{O}$ III] $\lambda 5007)$ " for our high redshift sample. We then show various new diagrams involving $L([\mathrm{O}$ III $]$ 15007), $L([\mathrm{O}$ II $] \lambda 3727)$ and $L(2-10 \mathrm{keV})$ and argue for new luminosity dependent correlations of narrow emission lines in type-II sources. Finally in Sect. 3 we discuss the new results, compare them with SDSS measurements of [O III] 15007 , and address some of the consequences to AGN LFs.

\section{X-ray and optical spectroscopy of new type-II AGNs}

\subsection{The new type-II AGN sample}

The new sample described in this paper is the result of a spectroscopic follow-up of the CDF-S. The spectroscopy and the selection criteria used to identify type-I and type-II AGNs, are explained in Szokoly et al. (2004). In short, we have obtained spectroscopic redshifts for 168 X-ray sources of which 137 have both reliable optical identification and redshift estimates. Out of them we identified 52 type-II AGNs based on their X-ray obscuring column and and luminosity (Tozzi et al. 2006a). To be fully consistent with the type-II definition, we only consider sources with $N_{\mathrm{H}}>10^{22} \mathrm{~cm}^{-2}$ and $L(2-10 \mathrm{keV})>10^{42}$ (the name "type-II QSOs" is reserved to those sources with $L(2-10 \mathrm{keV})>$ $\left.10^{44} \mathrm{erg} \mathrm{s}^{-1}\right)^{1}$. Two of those (CDF-S 202 and CDF-S 263) have already been discussed in previous papers (Norman et al. 2002; Mainieri et al. 2005a).

Table 1 summarizes all relevant spectroscopic data for the type-II sources belonging to this sample (for more spectroscopic information see Szokoly et al. 2004). Columns 1 and 2

1 An additional source, XID $=62$ from Giacconi et al. (2002), which would fit these selection criteria has been excluded since the optical spectroscopy reveals its BAL-QSO nature. give the object name and redshift and Cols. 6-14 give observed line fluxes for Ly $\alpha, \mathrm{C}$ IV $\lambda 1549$, He II $\lambda 1640, \mathrm{C}$ III] $\lambda 1909$, $\mathrm{Mg}$ II $\lambda 2798,\left[\mathrm{O}\right.$ II] $\lambda 3727, \mathrm{Ne}$ III $\lambda 3870, \mathrm{H} \beta$ and $\left[\mathrm{O}\right.$ III] $\lambda 5007^{2}$. Columns 3-5 provide information on the X-ray spectrum: the photon spectral index $\Gamma$, the obscuring neutral column $N_{\mathrm{H}}$ obtained from the fit and the intrinsic (corrected for absorption) 2-10 keV luminosity. These values have been obtained by a detailed spectral fitting procedure (Tozzi et al. 2006a) in which the default spectral model was a power law with slope $\Gamma$, intrinsic redshifted absorber with a neutral hydrogen column of $N_{\mathrm{H}} \mathrm{cm}^{-2}$, fixed Galactic absorption and an unresolved $6.4 \mathrm{keV} \mathrm{Fe} \mathrm{emission}$ line. We also allowed for the presence of a scattered component at soft energies with the same slope of the main power-law, and for a pure reflection typical of Compton-thick AGNs. We have decided to omit five Compton thick sources defined here as objects with $N_{\mathrm{H}}>10^{24} \mathrm{~cm}^{-2}$. These are likely to give highly uncertain, probably erroneous intrinsic $L_{[\mathrm{OIII}]} / L_{2-10}$.

The uncertainties on the X-ray measured fluxes can be obtained from Tozzi et al. (2006a). They are of the same order or smaller than the uncertainties associated with X-ray variability (about a factor 2). The uncertainties on the emission line measurements are typically $25 \%$. The combined uncertainty on the line to X-ray continuum, used later in our work, are therefore of order 2.

We have increased our sample size by including seven type-II QSOs from the spectroscopic follow-up of the HELLAS2XMM survey ${ }^{3}$. The X-ray spectral analysis for the additional sources has been performed by Perola et al. (2004) and the X-ray selection criteria fulfill those applied to the $\mathrm{CDF}-\mathrm{S}$ sample. The emission lines of these sources have been measured in a similar way to the other sample. Thus, the new X-ray selected sample includes 52 type-II AGNs out of which 18 are classified as type-II QSOs. All fluxes were converted to luminosities assuming a $\Lambda$-cosmology with $\Omega_{\mathrm{m}}=0.3, \Omega_{\Lambda}=0.7$ and $H_{0}=70 \mathrm{~km} \mathrm{~s}^{-1} \mathrm{Mpc}^{-1}$.

\subsection{Comparison with earlier type-II samples}

In order to compare our new results with previous works of this type, we made extensive use of the data in M 94, A97, Polletta et al. (1996) and Bassani et al. (1999). These references include $\mathrm{X}$-ray and [O III] $\lambda 5007$ fluxes for about 60 type-II mostly low $\mathrm{X}$-ray luminosity, low redshift sources. Unfortunately, some of the X-ray data in M 94 and in A97 were obtained with pre-ASCA instruments and the procedures used to obtain the $2-10 \mathrm{keV}$ absorption corrected luminosities suffer from various uncertainties. Moreover, the quality of the data did not allow the clear identification of Compton thick sources that are likely to bias the [O III] $\lambda 5007-X-$ ray comparison. Given this, we prefer to use the more uniform, carefully measured and modeled X-ray observations in Turner et al. (1997, 1998) and in Bassani et al. (1999). These are based on ASCA and BeppoSAX observations and considered to be more reliable. For example, a comparison of the Turner et al. results (see Table 12 in Turner et al. 1997) with M 94 shows a systematic trend for larger $L(2-10 \mathrm{keV})$ luminosities in the latter, especially for low luminosity X-ray sources. As shown below, this has important consequences to the main conclusion of M 94.

\footnotetext{
${ }^{2}$ Emission line fluxes for 7 of the sources are also shown in a paper by Nagao et al. that was submitted to publication after the submission of our paper - see arXiv: astro-ph/0508652.

3 The optical spectra are publicly available at: http://www. bo.astro.it/ hellas/sample.html
} 
Table 1. Emission line fluxes and X-ray properties of type-II AGNs.

\begin{tabular}{|c|c|c|c|c|c|c|c|c|c|c|c|c|c|}
\hline Object & $z$ & $\log L_{2-10}$ & $N_{\mathrm{H}}$ & $\Gamma_{2-10}$ & Ly $\alpha^{a}$ & C IV $\lambda 1549$ & He II $\lambda 1640$ & $\left.\mathrm{C}_{\text {III }}\right] \lambda 1909$ & Mg II $\lambda 2798$ & {$[\mathrm{O}$ II $] \lambda 3727$} & Ne III $\lambda 3870$ & $\mathrm{H} \beta$ & {$[\mathrm{O}$ III $] \lambda 5007$} \\
\hline CDF-S-10 & 0.424 & 42.66 & 1.5 & 1.17 & & & & & & 43.0 & & & \\
\hline CDF-S-18 & 0.979 & 44.05 & 1.9 & 1.74 & & & & & & 82.1 & & & \\
\hline CDF-S-20 & 1.016 & 43.27 & 5.6 & 1.78 & & & & & & 21.4 & & & \\
\hline CDF-S-27 & 3.064 & 44.33 & 28.1 & 1.22 & 12 & 6.2 & & & & & & & \\
\hline CDF-S-41 & 0.667 & 43.16 & 5.6 & 1.45 & & & & & & 122 & 46.4 & & 425 \\
\hline CDF-S-43 & 0.737 & 42.87 & 1.7 & 1.43 & & & & & & 9.18 & & & \\
\hline CDF-S-45 & 2.291 & 44.04 & 8.2 & 1.46 & 12.5 & & & & & & & & \\
\hline CDF-S-47 & 0.733 & 43.02 & 8.0 & 1.80 & & & & & & 22.7 & & & \\
\hline CDF-S-51 & 1.097 & 44.01 & 22.4 & 1.72 & & & & & & 36.7 & & & \\
\hline CDF-S-54 & 2.561 & 43.94 & 10.7 & 1.38 & 24.9 & 5.19 & & & & & & & \\
\hline CDF-S-56 & 0.605 & 43.31 & 1.6 & 1.25 & & & & & & 347 & & 190 & 841 \\
\hline CDF-S-57 & 2.562 & 44.20 & 19.3 & 1.69 & 109 & 16.6 & 5.66 & 12.2 & & & & & \\
\hline CDF-S-66 & 0.574 & 43.20 & 6.6 & 1.46 & & & & & & 34.6 & & & \\
\hline CDF-S-75 & 0.737 & 43.43 & 3.7 & 1.21 & & & & & & & & & 35.5 \\
\hline CDF-S-76 & 2.394 & 44.39 & 15.4 & 1.66 & 11.66 & & & & & & & & \\
\hline CDF-S-85 & 2.593 & 43.74 & 8.7 & 1.80 & 22.3 & & & & & & & & \\
\hline CDF-S-112 & 2.940 & 44.06 & 29.0 & 1.80 & 50.8 & 12.1 & 6.38 & & & & & & \\
\hline CDF-S-117 & 2.573 & 43.77 & 3.1 & 1.80 & 27.6 & & & & & & & & \\
\hline CDF-S-132 & 0.908 & 42.60 & 2.4 & 1.80 & & & & & & 6.97 & & & \\
\hline CDF-S-151 & 0.604 & 43.07 & 23.2 & 1.80 & & & & & & 14.2 & & & \\
\hline CDF-S-155 & 0.545 & 42.27 & 3.6 & 1.80 & & & & & & 66.1 & & 45.2 & 333 \\
\hline CDF-S-176 & 0.786 & 42.95 & 2.2 & 1.80 & & & & & & 16.4 & & & \\
\hline CDF-S-188 & 0.734 & 42.15 & 4.4 & 1.80 & & & & & & 8.44 & & & \\
\hline CDF-S-189 & 0.755 & 42.60 & 7.5 & 1.80 & & & & & & 5.44 & & & \\
\hline CDF-S-190 & 0.733 & 43.01 & 12.5 & 1.80 & & & & & & 125 & & & 176 \\
\hline CDF-S-201 & 0.679 & 42.59 & 2.6 & 1.80 & & & & & & 25.7 & 8.63 & & 114 \\
\hline CDF-S-204 & 1.223 & 42.43 & 7.5 & 1.80 & & & & & & 18.4 & & & \\
\hline CDF-S-252 & 1.172 & 43.21 & 15.8 & 1.80 & & & & & & 29.6 & & & \\
\hline CDF-S-260 & 1.043 & 43.01 & 36.7 & 1.80 & & & & & & 21.4 & & & \\
\hline CDF-S-264 & 1.316 & 43.23 & 21.6 & 1.80 & & & & & & 16.8 & & & \\
\hline CDF-S-266 & 0.735 & 43.33 & 88.8 & 1.80 & & & & & & 38.5 & & & 97.6 \\
\hline CDF-S-267 & 0.720 & 43.17 & 14.2 & 1.80 & & & & & 5.97 & & & & \\
\hline CDF-S-268 & 1.222 & 44.10 & 80.4 & 1.80 & & & & 13.1 & & & & & \\
\hline CDF-S-516 & 0.667 & 42.14 & 2.8 & 1.80 & & & & & & 39.0 & & & \\
\hline CDF-S-519 & 1.034 & 42.41 & 1.1 & 1.80 & & & & & & 17.7 & & & \\
\hline CDF-S-534 & 0.676 & 42.27 & 6.6 & 1.80 & & & & & & 16.0 & & & \\
\hline CDF-S-535 & 0.575 & 42.19 & 2.9 & 1.80 & & & & & & 50.0 & & & \\
\hline CDF-S-547 & 2.316 & 44.03 & 56.9 & 1.80 & 17.9 & & & & & & & & \\
\hline CDF-S-580 & 0.664 & 42.09 & 10.5 & 1.80 & & & & & & & & & 43.7 \\
\hline CDF-S-585 & 1.212 & 42.56 & 1.5 & 1.80 & & & & & & 13.8 & & & \\
\hline CDF-S-606 & 1.037 & 42.94 & 18.8 & 1.80 & & & & & & 9.39 & & & \\
\hline CDF-S-611 & 0.979 & 43.20 & 62.3 & 1.80 & & & & & & 11.2 & & & \\
\hline CDF-S-612 & 0.736 & 43.08 & 63.3 & 1.80 & & & & & & 23.7 & & & \\
\hline CDF-S-615 & 0.759 & 42.17 & 7.4 & 1.80 & & & & & & 11.7 & & & \\
\hline CDF-S-633 & 1.374 & 43.72 & 86.7 & 1.80 & & & & 31.3 & 18.0 & & & & \\
\hline H05370043 & 1.797 & 44.77 & 10.5 & 1.90 & & 119 & & & & & & & \\
\hline H05370164 & 1.824 & 44.48 & $0.0^{b}$ & 1.90 & & 16.6 & & & & & & & \\
\hline H05370016 & 0.995 & 44.35 & 1.3 & 1.90 & & & & & & 91.8 & & & \\
\hline H05370123 & 1.153 & 44.72 & 6.6 & 1.90 & & & & 51.6 & & 90.5 & & & \\
\hline H15800062 & 1.568 & 44.80 & 26.3 & 1.90 & & 51.6 & & 26.8 & & & & & \\
\hline H15800019 & 1.957 & 44.84 & 7.3 & 1.90 & & 451 & & & & & & & \\
\hline H50900013 & 1.261 & 44.55 & 2.5 & 1.90 & & & & 31.5 & & 70.1 & & & \\
\hline
\end{tabular}

${ }^{a}$ All line intensities are in units of $10^{-18} \mathrm{erg} \mathrm{s}^{-1} \mathrm{~cm}^{-2}$.

$\Gamma=1.80$ or $\Gamma=1.90$ means a guess of the $2-10 \mathrm{keV}$ slope.

Column density in units of $10^{22} \mathrm{~cm}^{-2}$.

$\mathrm{X}$-ray luminosities are corrected for intrinsic absorption.

${ }^{b}$ X-ray spectral parameters not available, classification based on optical spectrum.

The improved Turner et al. (1997) and Bassani et al. (1999) absorption corrected 2-10 keV luminosities were combined with the [O III] $\lambda 5007$ fluxes listed in M 94 and in Polletta et al. (1996) to obtain a high quality sample of low redshift type-II sources. Since there is much overlap between those lists, we chose as our primary source the Bassani et al. (1999) sample but carried a similar statistical analysis also for the Turner et al. data set with basically identical results. As explained earlier, we omit several Compton thick sources found in this sample. We have also excluded all objects with a noticeable Seyfert 1 contribution (we only include source listed as S1.8, S1.9 and S2), two AXJ sources (see Bassani et al. for justification) and sources 
with $z>0.05$. The result is a sample of 42 low redshift type-II AGNs. The cut in redshift is only to enable a clearer comparison with the new, high redshift sample. We have verified that the inclusion of the 6 sources omitted by the redshift criterion changes nothing for the statistical analysis that follows. For comparison we note that the original M 94 sample of type-II sources introducing the idea of a constant $L_{[\mathrm{OIII}]} / L_{2-10}$, includes only 16 low redshift objects.

The new high redshift sample requires a different procedure since it includes sources with various emission lines, mostly [O II] $\lambda 3727$, and only 8 measurement of the [O III] $\lambda 5007$ line intensity. However, there is enough information in the literature to enable the conversion from all those lines to [O III] $\lambda 5007$ intensity. We used a variety of data sets where UV as well as [O III] $\lambda 5007$ line fluxes are available. For Ly $\alpha$ in low redshift Seyfert 2s, we used ground-based observations of [O III] $\lambda 5007$ and IUE, HST and FUSE observations of the UV lines. Such data are very limited and the most useful sources are Ferland \& Osterbrock (1986) and Kuraszkiewicz et al. (2004). For this sample we find a mean of 0.81 and a median of 0.60 for $L(\mathrm{Ly} \alpha) / L([\mathrm{O}$ III] 15007$)$. There are also several extensive studies of narrow emission line galaxies, mostly radio loud high redshift galaxies, where rest-frame UV lines were observed from the ground, in the visual band, and the [O III] $\lambda 5007$ line was observed in the near IR, in the $H, J$ or $K$-bands. Work of this type is discussed in Evans (1998), Vernet et al. (2001), Stern et al. (1999), Rottgering et al. (1997), Stern et al. (2002) and Norman et al. (2002). Out of these references we found 11 objects that were considered appropriate to investigate the $I(\mathrm{Ly} \alpha) / I([\mathrm{O} \mathrm{III}]$ 25007) ratio in narrow line active galaxies. We found a mean of 2.40 and a median of 1.36 for the above line ratio in this group. Some of the new sources do not have measured Ly $\alpha$ and we had to use a similar method based on different UV lines, e.g. C IV $\lambda 1549$ or C III] $\lambda 1909$. All samples used to define these ratios are small and cover a large range in the properties under study. Therefore we prefer to use medians rather than means and a "typical range" which includes $2 / 3$ of the objects.

Our new sample contains 34 sources with measured [O II] $\lambda 3727$ line flux, thus the conversion to $L([\mathrm{O}$ III $] \lambda 5007)$ in this case is of a different nature since the final analysis will be dominated by those sources. We used the largest available sample where [O II] $\lambda 3727$ and [O III] $\lambda 5007$ luminosities are directly compared (291 sources in Zakamska et al. 2003). The mean $L([\mathrm{O} \mathrm{III}] \lambda 5007) / L([\mathrm{O} \mathrm{II}] \lambda 3727)$ in this sample is somewhat luminosity-dependent and is close to 3.5 over the range of $L([\mathrm{O} \mathrm{II}]$ 13727) found here. This is also close to the median of the 6 sources in our sample showing both [O II] $\lambda 3727$ and [O III] $\lambda 5007$ lines. Table 2 summarizes all these measurements by listing a composite type-II spectrum that was used to define an "effective" [O III] $\lambda 5007$ intensity for our high redshift sources. Many other composite spectra have appeared in the literature, e.g. Ferland \& Osterbrock (1986) and Zakamska et al. (2003). The most important results that are relevant here are that the median value of $I(\mathrm{Ly} \alpha) / I([\mathrm{O} \mathrm{III}] \lambda 5007)$ is very close to 1.0 and the one for $L([\mathrm{O} \mathrm{III}] \lambda 5007) / L([\mathrm{O} \mathrm{II}] \lambda 3727)$ is about 3.5 .

Having defined an effective $L([\mathrm{O} \mathrm{III}]$ 15007) from the observations of other emission lines, we can now compute $L_{[\mathrm{OIII}]} / L_{2-10}$ for all the new X-ray sources and compare them with the low redshift samples. This is shown in Fig. 1 where we display such ratios for the 52 new sources and also mark those where $L_{[\mathrm{OIII}]} / L_{2-10}$ is based on a conversion of a measured $L([\mathrm{O}$ II $]$ 13727). As seen from the diagram, there is a systematic trend in a sense that $L_{[\mathrm{OIII}]} / L_{2-10}$ is considerably smaller
Table 2. Composite type-II AGN spectrum.

\begin{tabular}{lcc}
\hline \hline Line & relative intensity & range \\
\hline$[\mathrm{O}$ III $] \lambda 5007$ & 1.0 & 1.0 \\
Ly $\alpha$ & 1.0 & $0.6-1.6$ \\
C IV $\lambda 1549$ & 0.30 & $0.2-0.5$ \\
He II $\lambda 1640$ & 0.15 & $0.1-0.4$ \\
C III $] \lambda 1909$ & 0.2 & $0.1-0.4$ \\
{$[\mathrm{O}$ II $] \lambda 3727$} & 0.29 & $0.2-0.8$ \\
\hline
\end{tabular}

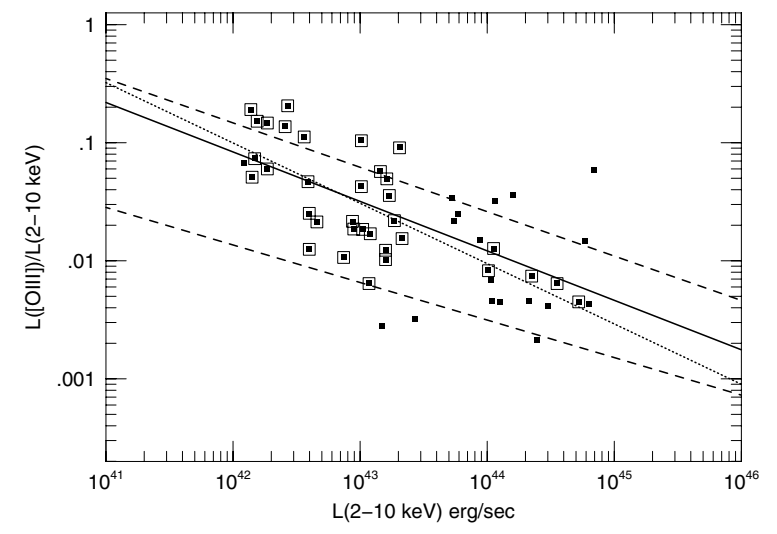

Fig. 1. $L_{[\mathrm{OIII}]} / L_{2-10}$ vs. $L(2-10 \mathrm{keV})$ for X-ray selected type-II AGNs. Full squares are data for the new high redshift sources using the "effective" [O III] $\lambda 5007$ luminosity as described in the text. Open squares are sources with measureable [O II] $\lambda 3727$ line. The solid line is a fit to the entire sample and the dotted line a fit to the [O II] $\lambda 3727$ sample only. Dashed lines are best fits to the Bassani et al. (1999) modified sample (see text). Lower curve: observed fluxes. Upper curve: line fluxes corrected for reddening.

for the higher X-ray luminosity sources. The statistical analysis confirms the high level of significance of the correlation. The Spearman rank correlation coefficient for the 52 sources is $r_{\mathrm{s}}=0.66\left(p=1.0 \times 10^{-7}\right)$ and a simple linear regression gives

$\log \frac{L_{[\mathrm{OIII}]}}{L_{2-10}}$ (type II uncorrected $)$
$=(16.5 \pm 2.9)-(0.42 \pm 0.07) \log L_{2-10}$

where we use "uncorrected" to indicate that the emission lines are not corrected for reddening. Since so many measurements are based on one line, we have also tested the correlation in the sub-sample of 34 sources with [O II] $\lambda 3727$ emission line. This also gives a very strong correlation $\left(r_{\mathrm{s}}=-0.70 p=4.9 \times 10^{-6}\right)$ with a somewhat steeper slope of $-(0.51 \pm 0.09)$. The slopes of the correlations are consistent within the errors. As evident from the diagram, the scatter is larger than the estimated uncertainties on individual points suggesting that there is a large range in the intrinsic properties of the sources. Finally we tested the correlation for the sub-sample of 24 sources showing emission lines other than [O II] $\lambda 3727$. This sub-sample shows a weaker but significant correlation $\left(r_{\mathrm{s}}=-0.57, p=0.003\right)$ with a slope of $-(0.39 \pm 0.14)$. For completion we note that in our new sample, $L(2-10 \mathrm{keV}) \propto L([\mathrm{O} \mathrm{III}] \lambda 5007)^{0.58}$, i.e. similar to the typical correlation between X-ray and UV continuum luminosities (e.g. Strateva et al. 2005).

Next we tested the 42 low redshift sources obtained from the Bassani et al. (1999) sample. The statistical analysis shows a weaker but significant correlation $\left(r_{\mathrm{s}}=-0.44, p=3.96 \times\right.$ $\left.10^{-3}\right)$ and a slope that is somewhat flatter $(-0.32 \pm 0.1)$ than the one found in the new sample. The best regression line is shown 
in Fig. 1. However, the mean $L_{[\mathrm{OIII}]} / L_{2-10}$ in this sample is considerably lower than the corresponding mean in the new sample (a factor of about 4 at $L(2-10 \mathrm{keV})=10^{44} \mathrm{erg} \mathrm{s}^{-1}$ ). As discussed later, we suspect that the difference is due to the way the sample was selected and the large amount of reddening (see Sect. 3.2). We also checked the low redshift data obtained from M 94, A97 and Turner et al. (1997) using only the more reliable X-ray data in Turner et al. The $L_{[\mathrm{OIII}]} / L_{2-10}$ vs. $L(2-10 \mathrm{keV})$ correlation is already present in this data set with a form very similar to what was found here. Thus, those correlations were present, yet never noticed, in older type-II AGN samples.

We note in passing two other sources of data for high luminosity type-II sources. Zakamska et al. (2004) identified six ROSAT all sky survey (RASS) type-II sources with measured [O III] $\lambda 5007$ and X-ray fluxes. As explained in their paper, the estimated $2-10 \mathrm{keV}$ flux is highly uncertain because of the limited ROSAT response at those energies and the likelihood of much absorption at low X-ray energies. Zakamska et al. suggest that the values of $L_{X}$ given in Table 4 of their paper are conservative lower bounds to the intrinsic 2-10 keV luminosities. Using those numbers we could obtain a conservative upper bounds of 0.013 for the median of $L_{[\mathrm{OIII}]} / L_{2-10}$ in this sample. This places the sources in the same region of Fig. 1 as our new objects with comparable X-ray luminosities. We prefer not to include these uncerain numbers in our statistical analysis. Ptak et al. (2005) list six new X-ray measurements for typeII SDSS sources. The $L_{[\mathrm{OIII}]} / L_{2-10}$ in this small groups shows a large scatter with a mean which is larger than the one found in our X-ray selected sample. As discussed below, this is likely to be attributed to a systematic difference between X-ray selected and optically selected samples and hence we do not include those AGNs in our analysis.

\section{3. $L_{[O I I I]} / L_{2-10}$ in type-I AGN}

To complete the description, we have constructed a similar ratio for type-I AGNs. There are many references for $L([\mathrm{O}$ III $]$ 15007) but fewer for $L(2-10 \mathrm{keV})$. Some are already included in M 94 and A97 who, at the time, did not have data on very high luminosity sources. There are also several detailed studies of the X-ray properties of low redshift quasars with measured $L([\mathrm{O}$ III $]$ 25007). These are mostly PG sources that were observed by ASCA and BeppoSAX and more recently by Chandra and XMM-Newton. Some of the data are given in George et al. (1998), Laor et al. (1998), Kaspi et al. (2005), Piconcelli et al. (2005) and Heckman et al. (2005). The most comprehensive of those is the Piconcelli et al. (2005) XMM-Newton data set. We note that corrections for intrinsic absorption at the $2-10 \mathrm{keV}$ range are very small in most of those sources except for a few (e.g. NGC 3783) that are known to have large column warm absorbers. Thus the main source of scatter is the intrinsic X-ray variability. We also note that the X-ray fluxes in Heckman et al. (2005) require a correction factor of 1.35 to take into account the somewhat different energy band (3-20 keV). The final sample contains 68 sources that are plotted in Fig. 2.

The statistical analysis of the type-I sample shows a strong correlation with $r_{\mathrm{s}}=-0.43\left(p=2.4 \times 10^{-4}\right)$. Again, this correlation was missed in the earlier M 94 and A97 works. The linear regression result is

$\log \frac{L_{[\mathrm{OIII}]}}{L_{2-10}}($ type I uncorrected $)$

$$
=(10.9 \pm 2.8)-(0.296 \pm 0.06) \log L_{2-10} .
$$

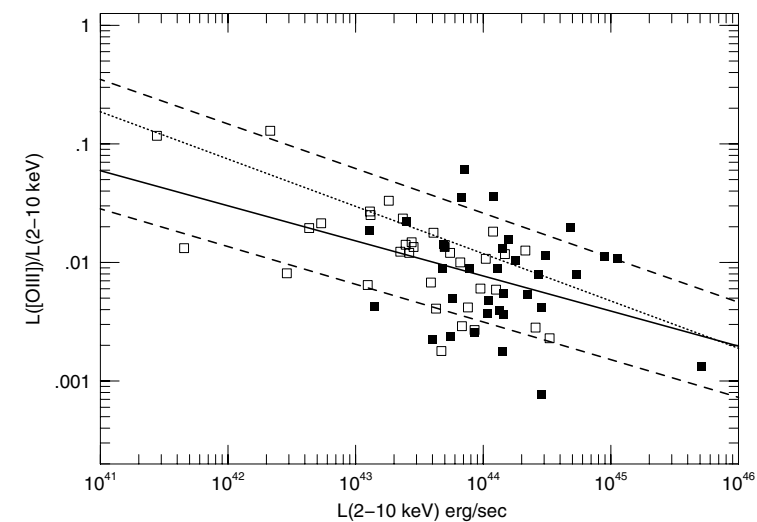

Fig. 2. Same as Fig. 1 but for type-I sources. Open symbols: data from M 94, A97 and Heckman et al. (2005). Full squares: data from BG92 and Piconcelli et al. (2005). The solid line is a linear fit to the data and the dotted line the fit to the type-II sample. Lower and upper dashed lines are the Bassani et al. (1999) curves shown in Fig. 1.

This relationship is plotted as a solid line in the diagram. We also show, as a dotted line, the relationship found for type-II sources as well as the two curves for the Bassani et al. (1999) type-II sample. As seen, the slopes of the correlations for the X-ray selected type-II sample and the optically selected typeI sample are somewhat different, but are consistent within $2 \sigma$. As argued in Sect. 3, this does not mean a similar behavior of type-I and type-II sources with regard to their optical vs. X-ray properties. For completeness, we have analyzed the Heckman et al. (2005) sample in the same way. We used the 34 type-I X-ray selected sources listed in this paper and found a very similar correlation to the one presented here. Heckman et al. discuss the linear relationship between $L(2-10 \mathrm{keV})$ and $L([\mathrm{O}$ III $] \lambda 5007)$ but do not comment on the luminosity dependence of $L_{[\mathrm{OIII}]} / L_{2-10}$. We must also note that $L([\mathrm{O} \mathrm{III}] \lambda 5007) / L([\mathrm{O}$ II $] \lambda 3727)$ in type-I sources is larger than in type-II sources (e.g. Zakamska et al. 2003; see also the special case of radio-loud sources in Jackson \& Brown 1990). This may introduce a systematic uncertainty when comparing our new sample (basically [O II] $\lambda 3727$ lines) with optically selected sample with measured [O III] $\lambda 5007$.

\section{Discussion}

Our new X-ray selected sample of 52 high redshift sources is the first of this type which is large enough to allow meaningful statistical analysis of the line-to-X-ray properties of type-II sources over a large luminosity range. The major findings of this work are strong dependences of $L_{[\mathrm{OIII}]} / L_{2-10}$ and $L_{[\mathrm{OII}]} / L_{2-10}$ on $L(2-10 \mathrm{keV})$, in type-II AGNs, contrary to past claims. Moreover, re-analysis of older data sets that do not include high luminosity, high redshift sources, also show a similar correlation. Our work shows a correlation of those properties in type-I AGNs, also in contrast to earlier claims.

The present analysis, as well as similar past works, is subjected to various uncertainties and biases. The two most important ones are related to sample selection and to narrow emission line reddening in AGNs.

\subsection{X-ray selected vs. optically selected AGN samples}

High quality X-ray spectroscopy of type-II sources with small to moderate obscuring columns can be used to reliably recover the 
intrinsic $L(2-10 \mathrm{keV})$ of the source. The only uncertainty is intrinsic continuum variablity which introduces a scatter of a factor $\sim 2$. This should not affect the correlation in large samples. More important is the difference between X-ray selected and optically selected samples. As discussed in many papers, most recently in Heckman et al. (2005), X-ray samples are biased towards X-ray bright sources and hence, in the case under study, will produce a smaller mean $L_{[\mathrm{OIII}]} / L_{2-10}$ compared to optically selected ones. While the Heckman et al. (2005) sample seems to confirm this suggestion, the data shown in Fig. 1 here seems to be in conflict with it since the mean $L_{[\mathrm{OIII}]} / L_{2-10}$ in our X-ray selected sample is larger than in the Bassani et al. (1999) sample. We suggest two reasons for this difference. One is line reddening which is discussed in Sect. 3.2 and the other is the difference between optical selection which is based on emission line strength (i.e. $E W$ ) and selection based on optical continuum flux.

Older samples of type-II sources were selected in various different ways. Some were found via direct spectroscopy of nearby bright emission line galaxies and others due to various other properties. This is clearly the case for most sources in M 94, A97, Bassani et al. (1999) and other older AGN samples. The SDSS is an $i$-mag selected sample and is hence biased towards high continuum flux type-I AGNs and strong emission line type-II AGNs. The former will be biased against large $E W$ lines while the later are expected to be biased against weak emission line sources. Thus, there are three possible biases to consider, two in optically selected samples and one in X-ray selected samples. Past, non-uniformally selected type-II sources that were found mostly in bright galaxies are not necessarily expected to show stronger $L_{[\mathrm{OIII}]} / L_{2-10}$ compared with sources in our new X-ray selected sample. On the other hand, we expect X-ray follow up on type-II SDSS sources to show larger $L_{[\mathrm{OIII}]} / L_{2-10}$. Given this, the comparison of past, randomly selected type-I and type-II samples is, perhaps, meaningful since many of those sources were discovered by the same techniques and are typical of optical continuum selected sources. More examples related to type-I SDSS sources are presented in Sect. 3.3.

\subsection{Emission line reddening}

Line reddening in type-II sources depends on the geometry and location of the NLR. A good example of the location dependent extinction is found in the recent work of Collins et al. (2005) on Mrk 3. Other detailed studies based on HST spectroscopy can be found in the literature (e.g. Ferruit et al. 1999). These few HST-based studies, combined with the IUE-based analysis of Ferland \& Osterbrock (1986), suggest that the narrow line reddening corresponds to $E(B-V)$ in the range $0.2-0.4$ mag. Other methods, based on the observed Balmer decrement, can be used to obtain the volume averaged narrow line reddening. For example, the median reddening found by Dahari \& De-Robertis (1988) in type-II sources is $E(B-V) \sim 0.54$ mag. These authors also mention that the (less certain) line reddening in type-I sources is smaller, by about $0.1-0.2$ mag. Bassani et al. (1999), following Maiolino et al. (1998), used various references to obtain $\mathrm{H} \alpha / \mathrm{H} \beta$ for all their sources. The median reddening in their sample is significantly larger, corresponding to $E(B-V) \sim 0.65 \mathrm{mag}$. More and independent confirmation of the large $\mathrm{H} \alpha / \mathrm{H} \beta$ in some of those Seyfert 2 galaxies can be found in Storchi-Bergmann et al. (1995). All those measurements give larger $E(B-V)$ than the mean $E(B-V) \sim 0.27$ mag. quoted by Zakamska et al. (2003) for their type-II SDSS AGN sample. The difference may be another manifestation of the bias against faint line type-II AGNs in the SDSS sample. This is likely to be more important at $z>0.2$, where the type-II SDSS sample is incomplete.

To quantify the effect of reddening, we have used the Bassani et al. (1999) lists to obtain the reddening corrected $L_{\text {[OIII }]} / L_{2-10}$ vs. $L(2-10 \mathrm{keV})$ for the 42 sources discussed in Sect. 2. We assumed an intrinsic $\mathrm{H} \alpha / \mathrm{H} \beta=3.1$ (e.g. Ferland \& Osterbrock 1986) and obtained

$$
\begin{aligned}
& \log \frac{L_{[\mathrm{OIII}]}}{L_{2-10}}(\text { type II corrected }) \\
& \quad=(15.0 \pm 4.0)-(0.38 \pm 0.09) \log L_{2-10}
\end{aligned}
$$

i.e. a significant correlation with a somewhat steeper slope than the one with no reddening. The shift in the mean $L_{[\mathrm{OIII}]} / L_{2-10}$ between the reddening corrected and the observed correlations, at $L(2-10 \mathrm{keV})=10^{44} \mathrm{erg} \mathrm{s}^{-1}$, is a factor of $\sim 8$. There is no tendency for more luminous X-ray sources to show more line reddening (which could have been an explanation for the decreasing $L_{\text {[OIII] }} / L_{2-10}$ with the X-ray luminosity).

Our new data contain mostly UV lines and we have no way to estimate reddening correction factors for individual sources. Regarding the mean properties of the sample, we note that the expected intensity ratio of $I(\mathrm{Ly} \alpha) / I([\mathrm{O} \mathrm{III}] \lambda 5007)$ is $\sim 9$. This number is obtained from combining the theoretical $I(\operatorname{Ly} \alpha) /(\mathrm{H} \beta) \simeq 50($ e.g. Netzer 1982; Ferland \& Osterbrock 1986) with the observed $I([\mathrm{O}$ III] $\lambda 5007) / I(\mathrm{H} \beta) \simeq 6$ (e.g. Zakamska et al. 2003 Table 2). This is a factor $\sim 9$ larger than the empirical ratio (Table 2) presented here and can be translated (assuming line of sight reddening) to galactic $E(B-V) \sim 0.3$ mag. Obviously, this average value is not very meaningful given the large scatter in $I(\mathrm{Ly} \alpha) / I([\mathrm{O} \mathrm{III}]$ 15007).

A related issue is the different mean $L_{[\mathrm{OIII}]} / L_{2-10}$ found between optically selected type-I and type-II samples. The optically selected samples studied here, albeit small and incomplete, show a clear shift in a sense that $L([\mathrm{O}$ III $]$ 15007) in type-I sources is larger, by a factor of $\sim 2$, for the same $L(2-10 \mathrm{keV})$. A possible explanation for the shift is additional reddening of [O III] $\lambda 5007$ in type-II sources. This can be the case if some of the reddening is due to a large structure, like the inner galactic disk, in disk-dominated systems with different orientations to the line-of-sight.

The results found here may look in conflict with studies of $I([\mathrm{O} \mathrm{III}] \lambda 5007) / I([\mathrm{O} \mathrm{II}] \lambda 3727)$ in type-I and type-II sources (Zakamska et al. 2003, and references therein). Such studies show this ratio to be larger in type-I sources despite the shorter wavelength of [O II] $\lambda 3727$, implying perhaps more reddening. However, the [O II] $\lambda 3727$ line emission region is much larger than the [O III] $\lambda 5007$ region and we do not expect the same amount of reddening in those two parts of the NLR. Other differences between type-I and type-II narrow line properties have been noted in the past (e.g. Zakamska et al. 2003, and references therein). Some of those may be related to similar geometrical factors. The conclusion is that the [O II] $\lambda 3727$ line luminosity may be a better measure of the NLR intrinsic emission and use of the $[\mathrm{O} \mathrm{III}] \lambda 5007$ flux should be treated with care.

Finally we comment on the fact that the $[\mathrm{O}$ III] $\lambda 5007$ emission is known to depend on radio properties being stronger in radio-loud sources. We have $20 \mathrm{~cm}$ radio VLA data for all sources in our sample (Kellerman et al. 2006, in preparation). Only 3 were detected to a flux limit of $42 \mu \mathrm{Jy}$. Thus, radio properties cannot influence much the present results. 


\section{3. $L([O I I I] \lambda 5007)$ as a luminosity indicator in AGNs}

The correlations found here allow us to investigate the use of $L([\mathrm{O} \mathrm{III}]$ 25007) as a luminosity indicator in AGNs. For this we look for the correlation of the equivalent width of this line with the optical continuum, $L_{5100}$, defined here as the uncorrected $\lambda L_{\lambda}$ at $5100 \AA$. To obtain an expression for the expected $E W([\mathrm{O}$ III $]$ 15007) we assume a $0.1-1 \mu$ m power-law continuum with an optical energy slope of $\alpha_{\mathrm{op}}=0.5$ and a hard X-ray (2-10 keV) energy slope of $\alpha_{\mathrm{x}}=0.9$. Given this spectral energy distribution (SED), and $\alpha_{\text {ox }}$ (the energy slope connecting $2500 \AA$ and $2 \mathrm{keV})$, we find $\log \left(L_{2-10} / L_{5100}\right)=3-2.605 \alpha_{\text {ox }}$. We can thus write the following expression for $E W([\mathrm{O}$ III $] \lambda 5007)$,

$\log (E W([\mathrm{OIII}]]))=6.7-2.605 \alpha_{\text {ox }}+\log \frac{L_{[\mathrm{OIII}]}}{L_{2-10}}$,

which again assumes no reddening. A specific $E W([\mathrm{O}$ III $] \lambda 5007)$ can be obtained by taking into account the known dependence of $\alpha_{\mathrm{ox}}$ on UV and optical luminosity (Strateva et al. 2005).

Data on $E W([\mathrm{O} \mathrm{III}]$ 15007) in very large samples are now available from the $2 \mathrm{dF}$ and the SDSS samples. This line is relatively easy to measure in luminous type-I sources and work of this type has been published in several papers (e.g. S05; Hao et al. 2005). We have made our own study of the SDSS archive using basically all type-I sources available at this stage with $z<0.75$. A detailed description of the procedure used to measure all emission lines will be given elsewhere (Netzer \& Trakhtenbrot 2006). In brief, we have used the fourth data release (Adelman-McCarthy et al. 2006) and included only sources within the magnitude limit of this sample ( $i=19.1 \mathrm{mag}$ ). We measured the [O III] $\lambda 5007$ line flux and $E W$ by using single Gaussian fits to the two [OIII] lines and by employing a sophisticated procedure to deblend and subtract the strong FeII lines over the 4400-5500 $\AA$ range. This underestimates, somewhat, the real [O III] $\lambda 5007$ intensity since the line is known to have an extended blue wing in many AGNs (Greene \& Ho 2005, and references therein). However, the single-component fit is more robust, especially in type-I sources because of the easier de-blending with the broad $\mathrm{H} \beta$ profile. It also provides a more consistent way for comparison with type-II SDSS sources that were mostly measured in a similar way. Some 2000 sources were removed from the analysis mostly because they did not fulfill the AGN detection criteria and because of observational uncertainties (see Netzer \& Trakhtenbrot 2006, for more details). We chose not to remove those $\sim 10 \%$ of the sources that are radio loud but verified that none of the conclusions discussed below are affected by their inclusion. The end result is a sample of about 12000 sources with measured intensities or upper limits on the [O III] $\lambda 5007$ line.

The SDSS is a flux limited sample and hence suffers from various selection effects, especially near its flux limit. As explained earlier, this may affect the $E W$ distribution of [O III] $\lambda 5007$ and other emission lines since source selection is based on continuum SED (or "colors") and continuum flux (or broad band magnitudes). Because of this, we have investigated, separately, the luminosity dependent and the redshift dependent distributions of $E W([\mathrm{O} I I I] \lambda 5007)$. The results are shown in Fig. 3 where the median $E W([\mathrm{O} \mathrm{III}]$ 25007) is plotted in various ways. The diagram shows a tendency for $E W([\mathrm{O}$ III $] \lambda 5007)$ to decrease as a function of $L_{5100}$ at $z>0.3$ but there is no obvious global redshift dependence. Adding all redshift bins gives a change of the median $E W$ by a factor of about 1.7 from $L_{5100}=$ $10^{43} \mathrm{erg} \mathrm{s}^{-1}$ to $L_{5100}=10^{46} \mathrm{erg} \mathrm{s}^{-1}$. The mean $E W([\mathrm{O} \mathrm{III}] \lambda 5007)$

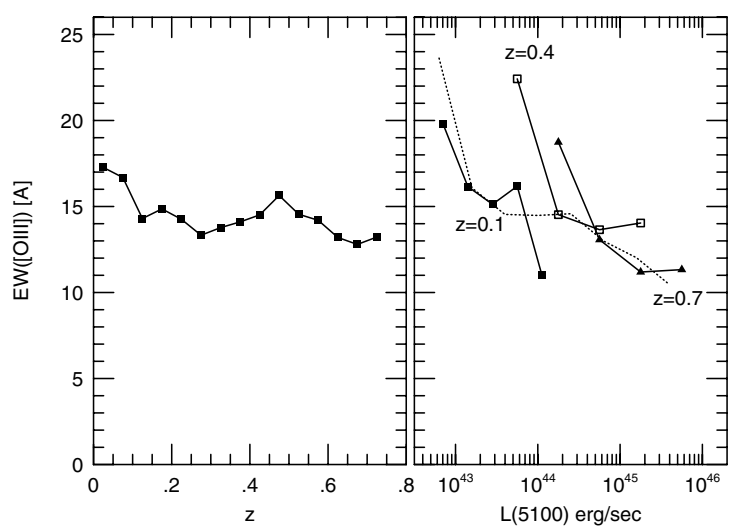

Fig. 3. The median $E W([\mathrm{O} \mathrm{III}] \lambda 5007)$ as a function of redshift (left) and continuum luminosity $\left(\lambda L_{\lambda}\right.$ at $5100 \AA$, right $)$ for 11900 type-I SDSS AGNs. The luminosity dependence is shown for three different redshift bands (solid lines with symbols) and the entire sample (dotted line).

(not shown here) has a similar trend with $L_{5100}$ and is larger than the median by a factor of about 1.5 .

The tendency for $E W([\mathrm{O} \mathrm{III}] \lambda 5007)$ to decrease with continuum luminosity (the "Baldwin effect", see Baldwin 1977) has been studied in previous work including in Croom et al. (2002) and in Netzer et al. (2004). The Croom et al. study includes several thousands $2 \mathrm{dF}$ AGNs with measured [O III] $\lambda 5007$ line in the redshift range $0-0.5$. The sample includes sources that are almost a magnitude fainter than the faintest SDSS AGNs. According to this paper, there is no Baldwin relationship for this line. In fact, the line $E W$ tends to increase somewhat with increasing luminosity. The Netzer et al. (2004) sample includes some of the most luminous high redshifts QSOs. According to these authors, the Baldwin effect seen in previous [O III] $\lambda 5007$ samples is not due to a decreasing mean line luminosity but rather the larger fraction of high luminosity AGNs with extremely week, sometimes unobservable [O III] $\lambda 5007$ lines. According to these authors, some very high luminosity AGNs show large $E W([\mathrm{O}$ III $]$ 15007), similar to the one observed in lower luminosity sources, but others show a very weak line. In such cases, the mean of the population may not be a very meaningful quantity since we may be dealing with a bimodal distribution of equivalent widths. In the Netzer et al. sample, the luminosity is more than an order of magnitude larger than the highest luminosity considered in the present work and about a third of the sources have undetectable [O III] $\lambda 5007$ lines.

The analysis of the 12000 type-I SDSS AGNs seem to be consistent with both Croom et al. (2002) and Netzer et al. (2004). While there seem to be a trend in both median and mean at all redshifts (Fig. 3), this is not statistically significant at the low luminosity, low redshift end. This has been verified by a regression analysis of the lower redshift sources. We find that in the range $0<z<z_{1}$, where $z_{1}<0.3$, there is no significant Baldwin effect while at higher redshifts, where the mean $L$ ([O III] $\lambda 5007$ ) is larger, there is a significant effect. The Croom et al. (2002) $2 \mathrm{dF}$ sample includes lower luminosity lower redshift sources and the lack of a Baldwin effect in their data is in agreement with our finding. The trend at larger luminosities and redshifts in our sample is statistically significant and the mean $E W([\mathrm{O}$ III $]$ 25007) is indeed decreasing with $L_{5100}$. Some of it, especially at the largest $z$ considered here, may be related to the flux limit of the SDSS sample. Unfortunately, we are not in a position to investigate in detail the relationship between $E W([\mathrm{O}$ III $]$ 15007) 
and $L_{[\mathrm{OIII}]} / L_{2-10}$ in optically selected type-I sources because of the small number of available $L(2-10 \mathrm{keV})$ measurements. However, it is obvious that $L_{[\mathrm{OIII}]} / L_{2-10}$ found here and shown in Fig. 1, drops faster with $L(2-10 \mathrm{keV})$ compared with the drop of $E W\left([\mathrm{O}\right.$ III $]$ 25007) with $L_{5100}$ (note that the drop of $\alpha_{\text {ox }}$ with optical and UV continuum luminosity causes an opposite effect).

Given Eq. (4) we can work out the expected (i.e. as would be seen against an unobscured continuum) $E W([\mathrm{O}$ III $]$ 15007) in our type I and type-II samples. Assume $\alpha_{\mathrm{ox}}=1.4$ and $L(2-10 \mathrm{keV})=10^{44} \mathrm{erg} \mathrm{s}^{-1}$ (see Fig. 2). This would give $L_{[\mathrm{OIII}]} / L_{2-10}=7.5 \times 10^{-3}$ (Eq. (2)) and hence $E W([\mathrm{O}$ III $] \lambda 5007) \simeq 9 \AA$, close to the median at the high luminosity end in Fig. 3. Thus, except for an obvious difference between the median and the mean, the high luminosity type-I X-ray sources studied here have typical values of $E W([\mathrm{O}$ III $]$ 15007) similar to those observed at the high luminosity end of our $z<$ 0.75 SDSS sample. For comparison, the Heckman et al. (2004) work on type-II sources assume a typical $L([\mathrm{O}$ III] 25007$)$ which is equivalent to $E W([\mathrm{O}$ III $] \lambda 5007) \simeq 15 \AA$.

Finally we comment on the expected number of high luminosity AGNs with detectable emission lines. The mean (uncorrected) $L([\mathrm{O} \mathrm{III}]$ 25007) observed at the highest luminosity end in Fig. 1 can be translated (see Eq. (4)) to $E W([\mathrm{O}$ III $]$ 15007) < $6 \AA$. At higher luminosities, like those found in the Netzer et al. (2004) sample, the expected $E W([\mathrm{O}$ III $]$ 25007) is even smaller and $E W([\mathrm{O} \mathrm{II}]$ 13727) is about a factor of 5 below this number. Such equivalent widths are close to the detection limit of many spectroscopic surveys. These numbers suggest that many objects with very high X-ray luminosities, would have extremely weak emission lines and would not be detected by spectroscopic follow ups of optically selected and X-ray selected samples (see the Netzer et al. (2004) more detailed discussion of this point).

A recent paper by Martinez-Sansigre (2005) discusses a sample of 21 type-II high luminosity AGNs discovered by their radio and infrared properties in the Spitzer first look survey (SLF). Deep spectroscopic follow-up resulted in narrow emission line detection of 10 of the sources. Eleven others, while being of similar mid-IR properties, did not show a trace of any emission line. According to the paper, the sources without detected narrow lines are those where galactic scale obscuration increased the line reddening of the otherwise normal NLRs. We suggest that the lack of detected narrow lines in AGNs found in such infrared surveys is related to the present finding of extremely weak emission lines in X-ray detected AGNs. For example, the analysis of our own spectroscopic follow-up (Szokoly et al. 2004) shows that out of 106 type-II candidates selected by the column density and the X-ray luminosity (based on photo- $z$ ) we could only obtain redshifts for 69 . The remaining 37 sources $(35 \%)$ show no emission lines. These sources have X-ray luminosities similar to the ones presented here and the integration times, and overall observing conditions, were very similar to those used to find the sources listed in Table 1.

All the above findings are consistent with the Netzer et al. (2004) "disappearing NLR" suggestion that a large fraction of high luminosity AGNs of both types show extremely weak narrow emission lines. One explanation is that in many such sources, the NLR size exceeds the galactic size and most of the gas escapes the system. Since the [O II] $\lambda 3727$ emission region is considerably larger than the [O III $] \lambda 5007$ zone, we expect the effect to be more pronounced in the former case, i.e. a steeper dependent of $L_{[\mathrm{OII}]} / L_{2-10}$ on $L(2-10 \mathrm{keV})$ compared with $L_{[\mathrm{OIII}]} / L_{2-10}$, at the high luminosity end. Such extreme type-II sources will never be found by their emission lines.
Another possibility, is extremely large emission line reddening preferentially in the highest luminosity sources but the data presented here do not support this view. All this must be taken into account when assessing the type-II contribution to the $\mathrm{CXB}$, using emission line surveys.

\subsection{Implications to AGN Iuminosity function and space distribution}

AGN LFs have been the subject of much discussion in recent years, following the publication of several large new systematic surveys like $2 \mathrm{dF}$ and SDSS. Such surveys allow a comparison of various properties as a function of source luminosity, redshift and even black hole mass. Comprehensive studies, and many references can be found in Croom et al. (2004), Hao et al. (2005) and S05.

Measured emission line fluxes in thousands of SDSS sources, allowed Hao et al. (2005a,b) and S05 to construct emission line LFs for the [O III $] \lambda 5007$ line. The line flux in these studies was assumed to be independent of orientation and thus a good measure of the intrinsic source luminosity. No reddening correction was used when deriving the LF. Hao et al. (2005b) investigated sources with $0<z<0.15$ with the conclusion that the two AGN types are indistiguisable at low luminosity but type-I sources outnumber type-II ones at the high luminosity end. S05 used a somewhat different redshift range (0.02-0.3) and claimed a more sever paucity of type-II sources at large [O III] $\lambda 5007$ luminosity. S05 further compared the results with the prediction of the "receding torus" model. According to this model, the opening angle of the central obscuring torus is luminosity dependent and higher luminosity sources have larger openings. This suggests that the fraction of type-I sources would be larger at the high luminosity end. More details of the model can be found in Lawrence (1991), Simpson (1998) and Grimes et al. (2004).

The empirical [O III] $\lambda 5007 \mathrm{LF}$ found by S05 is in contradiction to the simple receding torus model and the paper proceeds to examine the various possibilities for the discrepancy. In particular it shows that some of the discrepancy can be explained by neglecting the assumption of a luminosity independent $L_{[\mathrm{OIII}]} / L_{2-10}$. Specifically, a relationship of the type

$$
L([\mathrm{OIII}] \lambda 5007) \propto L_{\mathrm{rad}}^{1-2 \xi}
$$

where $L_{\mathrm{rad}}$ is the total radiated continuum luminosity that heats the dust in the torus, with $\xi \simeq 0.23$, can lead to a much better agreement between model and observations. The author then dismisses this idea on grounds of the well known near-constancy of $E W([\mathrm{O} \mathrm{III}]$ 15007) in QSOs based on the Miller et al. (1992) results and the known proportionalities between $L([\mathrm{O}$ III $]$ 15007) and other quantities based on M 94 and Grimes et al. (2004). As shown here, some of those early claims are not substantiated by our new analysis of X-ray selected type-II and optically selected type-I AGN samples. In fact, our Fig. 1 and Eqs. (1) and (2) show relationships of exactly the type suggested by S05. Thus part of the discrepancy can be explained in this way. This cannot be the entire explanation since our data also show a shift in the mean $L_{[\mathrm{OIII}]} / L_{2-10}$ between the two AGN groups. We suggest that the $\mathrm{S} 05$ calculations be repeated taking into account all those effects, including the possibility of disappearing NLRs at high continuum luminosities. A full investigation of this type is beyond the scope of the present paper. 
Acknowledgements. We are grateful to R. Maiolino, G. Szokoly, M. Crenshaw, S. Kraemer, G. Hasinger, J. Turner, G. Kauffmann and Marcella Brusa for helpful comments and discussions. We also benefitted from the useful comments of the referee, M. Strauss. Funding for the creation and distribution of the SDSS Archive has been provided by the Alfred P. Sloan Foundation, the Participating Institutions, the National Aeronautics and Space Administration, the National Science Foundation, the U.S. Department of Energy, the Japanese Monbukagakusho, and the Max Planck Society. The SDSS Web site is http://www.sdss.org/. The SDSS is managed by the Astrophysical Research Consortium (ARC) for the Participating Institutions. The Participating Institutions are The University of Chicago, Fermilab, the Institute for Advanced Study, the Japan Participation Group, The Johns Hopkins University, Los Alamos National Laboratory, the Max-Planck-Institute for Astronomy (MPIA), the Max-Planck-Institute for Astrophysics (MPA), New Mexico State University, University of Pittsburgh, Princeton University, the United States Naval Observatory, and the University of Washington. This research has made use of the NED database which is operated by the Jet Propulsion Laboratory, California Institute of Technology, under contract with the National Aeronautics and Space Administration. This work is supported by the Israel Science Foundation grant 232/03 and by the chair of Extragalactic Astronomy at Tel Aviv University. HN acknowledges an Humboldt foundation prize and thank the host institution, MPE Garching, where most of this work has been done.

\section{References}

Adelman-McCarthy, J. K., Jennifer, K., Agüeros, M. A., et al. 2006, ApJS, 162, 38

Alonso-Herrero, A., Ward, M. J., \& Kotilainen, J. K. 1997, MNRAS, 288, 977 (A97)

Baldwin, J. A. 1977, ApJ, 214, 679

Bassani, L., Dadina, M., Maiolino, R., et al. 1999, ApJS, 121, 473

Boroson, T. A., \& Green, R. F. 1992, ApJS, 80, 109 (BG92)

Collins, N. R., Kraemer, S. B., Crenshaw, D. M., et al. 2005, ApJ, 619, 116

Croom, S. M., Rhook, K., Corbett, E. A., et al. 2002, MNRAS, 337, 275

Dahari, O., \& de Robertis, M. M. 1988, ApJS, 67, 249

Ferruit, P., Wilson, A. S., Whittle, M., et al. 1999, ApJ, 523, 147

Fiore, F., Brusa, M., Cocchia, F., et al. 2003, A\&A, 409, 79

George, I. M., Turner, T. J., Netzer, H., et al. 1998, ApJS, 114, 73

Giacconi, R., Zirm, A., Wang, J.-X., et al. 2002, ApJS, 139, 369

Giavalisco, M., Ferguson, H. C., Koekemoer, A. M., et al. 2004, ApJ, 600, L93

Greene, J. E., \& Ho, L. C. 2005, ApJ, 627, 721

Grimes, J. A., Rawlings, S., \& Willott, C. J. 2004, MNRAS, 349, 503
Heckman, T. M., Kauffmann, G., Brinchmann, J., et al. 2004, ApJ, 613, 109

Heckman, T. M., Ptak, A., Hornschemeier, A., \& Kauffmann, G. 2005 [arXiv:astro-ph-0507674]

Hao, L., Strauss, M. A., Fan, X., et al. 2005, AJ, 129, 1795

Jackson, N., \& Browne, I. W. A. 1990, Nature, 343, 43

Kaspi, S., Maoz, D., Netzer, H., et al. 2005, ApJ, 629, 61

Kellerman, et al. 2006, ApJ, submitted

Kuraszkiewicz, J. K., Green, P. J., Crenshaw, D. M., et al. 2004, ApJS, 150, 165

Lawrence, A. 1991, MNRAS, 252, 586

Mainieri, V., Rosati, P., Tozzi, P., et al. 2005a, A\&A, 437, 805

Mainieri, V., Rigopoulou, D., Lehmann, I., et al. 2005b, MNRAS, 356, 1571

Maiolino, R., Salvati, M., Bassani, L., et al. 1998, A\&A, 338, 781

Martínez-Sansigre, A., Rawlings, S., Lacy, M., et al. 2005, Nature, 436, 666

Miller, P., Rawlings, S., Saunders, R., \& Eales, S. 1992, MNRAS, 254, 93

Mulchaey, J. S., Koratkar, A., Ward, M. J., et al. 1994, ApJ, 436, 586 (M 94)

Nagao, T., Maiolino, R., \& Marconi, A. [arXiv:astro-ph/0508652]

Netzer, H., Shemmer, O., Maiolino, R., et al. 2004, ApJ, 614, 558

Netzer, H., \& Trakhtenbrot, B., 2006, ApJ, submitted

Norman, C., Hasinger, G., Giacconi, R., et al. 2002, ApJ, 571, 218

Perola, G. C., Puccetti, S., Fiore, F., et al. 2004, A\&A, 421, 491

Piconcelli, E., Jimenez-Bailón, E., Guainazzi, M., et al. 2005, A\&A, 432, 15

Polletta, M., Bassani, L., Malaguti, G., Palumbo, G. G. C., \& Caroli, E. 1996, ApJS, 106, 399

Ptak, A., Zakamska, N., Strauss, M., et al. 2006, ApJ, in press [arXiv: astro-ph/0510204]

Rosati, P., Tozzi, P., Giacconi, R., et al. 2002, ApJ, 566, 667

Simpson, C. 2005, MNRAS, 360, 565 (S05)

Yuan, J., \& Dietrich, M. 2003, ApJ, 583, 124

Storchi-Bergmann, T., Kinney, A. L., \& Challis, P. 1995, ApJS, 98, 103

Turner, T. J., George, I. M., Nandra, K., \& Mushotzky, R. F. 1997, ApJS, 113, 23

Turner, T. J., George, I. M., Nandra, K., \& Mushotzky, R. F. 1998, ApJ, 493, 91 Stern, D., Moran, E. C., Coil, A. L., et al. 2002, ApJ, 568, 71

Strateva, I. V., Brandt, W. N., Schneider, D. P., Vanden Berk, D. G., \& Vignali, C. $2005, \mathrm{AJ}, 130,387$

Szokoly, G. P., Bergeron, J., Hasinger, G., et al. 2004, ApJS, 155, 271

Tozzi, et al. 2006a, A\&A, in press [arXiv: astro-ph/0602127]

Tozzi, P., Gilli, R., Mainieri, V., et al. 2006b, ApJ, submitted

Vignali, C., Alexander, D. M., \& Comastri, A. 2004, MNRAS, 354, 720

Zakamska, N. L., Strauss, M. A., Krolik, J. H., et al. 2003, AJ, 126, 2125

Zakamska, N. L., Strauss, M. A., Heckman, T. M., Ivezić, Ž., \& Krolik, J. H. 2004, AJ, 128, 1002 